\title{
Dichloroacetate prevents but not reverses the formation of neointimal lesions in a rat model of severe pulmonary arterial hypertension
}

\author{
BINGBING LI $^{1 *}$, JIE YAN $^{2 *}$, YAN SHEN $^{1}$, YONG LIU $^{3}$ and ZHENGLIANG MA ${ }^{1}$ \\ ${ }^{1}$ Department of Anesthesiology, Nanjing Drum Tower Hospital, The Affiliated Hospital of Nanjing University Medical School, \\ Nanjing, Jiangsu 210008; ${ }^{2}$ The State Key Laboratory of Reproductive Medicine, Department of Anesthesiology, \\ The Nanjing Maternity and Child Health Care Hospital Affiliated with Nanjing Medical University, Nanjing, Jiangsu 210004; \\ ${ }^{3}$ Jiangsu Key Laboratory for Molecular Medicine, Nanjing University Medical School, Nanjing, Jiangsu 210008, P.R. China
}

Received October 4, 2013; Accepted April 24, 2014

DOI: $10.3892 / \mathrm{mmr} .2014 .2432$

\begin{abstract}
The formation of neointimal lesions is one of the characteristic pathological alterations of the pulmonary vasculature in response to sustained pulmonary arterial hypertension (PAH). To date, the effect of dichloroacetate (DCA) on these intimal lesions had remained to be investigated. In the present study, the beneficial impact of DCA on the formation of neointimal lesions was examined in rats treated with monocrotaline following pneumonectomy. The rats were subjected to monocrotaline subcutaneous injection seven days following left pneumonectomy and received DCA by gastric gavage $(80 \mathrm{mg} / \mathrm{kg} / \mathrm{day})$ for 20 days. At the end of the experiments, hemodynamic measurement was performed prior to the excision of the rats' right lungs for further morphometric and immunoblot analysis. Furthermore, the activity of reactive oxygen species (ROS), superoxide dismutase (SOD) in
\end{abstract}

Correspondence to: Professor Zhengliang Ma, Department of Anesthesiology, Nanjing Drum Tower Hospital, The Affiliated Hospital of Nanjing University Medical School, Zhongshan Road 321, Nanjing, Jiangsu 210008, P.R. China

E-mail:041104160@fudan.edu.cn

*Contributed equally

Abbreviations: $\mathrm{Cu} / \mathrm{Zn}$ SOD, copper/zinc superoxide dismutase; DCA, dichloroacetate; DMSO, dimethyl sulfoxide; HIF-1 $\alpha$, hypoxia inducible factor $\alpha$ : MCT, monocrotaline; MnSOD, manganese superoxide dismutase; PAH, pulmonary arterial hypertension; PAP, pulmonary arterial pressure; PBS, phosphate-buffered saline; PDH, pyruvate dehydrogenase; ROS, reactive oxygen species; RV/LV+IVS, right ventricle/left ventricle+intra-ventricle septum; RVSP, right ventricle systolic pressure; Kv1.5, voltage-dependent potassium channel subtype 1.5

Key words: pulmonary arterial hypertension, intimal lesion formation, dichloroacetate, hypoxia inducible factor, superoxide dismutase the lungs was examined by a colorimetric assay. The results revealed that DCA treatment from day 8-28 following pneumonectomy caused a marked reduction in pulmonary arterial pressure and amelioration in right ventricle hypertrophy (mean pulmonary arterial pressure, $24 \pm 2.8$ vs. $33 \pm 5.5 \mathrm{mmHg}$; right ventricle-to-left ventricle + intra-ventricle septum ratio, $29 \pm 2.8$ vs. $43 \pm 3.3 \% ; \mathrm{P}<0.05)$ and pulmonary arterioles intimal proliferation (grade 1 and 2 occlusion, 24 and $20 \%$ vs. 44 and $40 \%$; $\mathrm{P}<0.05)$ in pulmonary hypertensive rats. By contrast, DCA treatment initiated 29 days following pneumonectomy did not result in any improvement in pulmonary circulatory parameters or regression of occlusive neointimal lesions. Immunohistochemical and immunoblot assays demonstrated markedly reduced hypoxia inducible factor (HIF-1 $\alpha$ ) levels, and increased voltage-dependent potassium channel subtype 1.5 (Kv1.5) expression levels were observed in those neointimal regions in rats receiving DCA preventive therapy. Furthermore, preventive treatment with DCA significantly increased the activity of copper/zinc superoxide dismutase $(\mathrm{Cu} / \mathrm{Zn}$ SOD activity, $88 \pm 4.2$ vs. $53 \pm 2.7 \mathrm{U} / \mathrm{mgprot}$; $\mathrm{P}<0.05)$ and promoted the degradation of ROS $(106 \pm 4.7$ vs. $79 \pm 13.3 \mathrm{U} / \mathrm{mgprot} ; \mathrm{P}<0.05)$, which was compromised in the delayed intervention group. Therefore, DCA is effective to prevent the formation of intimal lesions, which may be attributed to the induction of the upregulation of $\mathrm{Cu} / \mathrm{Zn}$ SOD activity and the suppression of HIF-1 $\alpha$ activation.

\section{Introduction}

Pulmonary arterial hypertension (PAH), a group of diseases defined as a mean pulmonary arterial pressure of $>25 \mathrm{mmHg}$ at rest with an pulmonary arterial wedge pressure of $<15 \mathrm{mmHg}$, occurs with an idiopathic aetiology or in association with diseases including congenital heart malformation, connective tissue disease or human immunodeficiency virus infection (1). PAH is essentially a vasculopathy in the pulmonary circulation, manifesting narrowing or occlusion of the vessel lumen in pre-capillary arterioles due to the formation of neointimal or plexiform lesions. Despite extensive evidence for the mechanisms underlying the pathophysiological etiology 
of PAH, few effective therapeutic modalities have emerged as a result $(2,3)$.

Dichloroacetate (DCA) is as a metabolism modulator, which has been utilized for approximately three decades as the first line drug in the treatment of diseases associated with mitochondrial dysfunction, including congenital lactic acidosis (4). Several previous studies indicated that DCA may reverse the medial thickening in the monocrotaline (MCT) model, hypoxic PAH in rats and SM22 $\alpha$-targeted over-expression of the serotonin transporter in mouse models (5-7). The mechanism underlying this effect may be the inactivation of pyruvate dehydrogenase $(\mathrm{PDH})$ kinase and transportation of pyruvate into the Krebs cycle instead of lactic production, which leads to depolarization of the mitochondrial membrane potential and initiation of apoptosis in pulmonary arterial smooth muscle cells (SMCs) (8). However, the efficacy of DCA in MCT-treated pneumonectomized rats is yet to be investigated. DCA was identified to be effective in promoting apoptosis in a variety of cancer cell lines $(9,10)$. Furthermore, the treatment of epithelial ovarian cancer cells with DCA markedly increased superoxide dismutase (SOD) transcription and protein expression levels and ultimately resulted in the inactivation of hypoxia-inducible factor- $1 \alpha(\mathrm{HIF}-1 \alpha)$ and activation of the mitochondrial apoptosis pathway (11).

Hypoxia-inducible factor-1 (HIF-1), a heterodimer of HIF- $1 \alpha$ and HIF-1 $\beta$, regulates the transcriptional activation of genes involved in energy metabolism, vasomotor tone and angiogenesis, as part of an adaptive molecular response to low oxygen availability (12). Several previous studies suggested that HIF-1 $\alpha$ activation was involved in angiogenesis in patients with PAH complicated with congenital heart malformation or idiopathic forms other than hypoxia-induced PAH (13). Consistent with this, a previous study by our group demonstrated that HIF-1 $\alpha$ levels were markedly increased in the neointimal lesion areas in pneumonectomized rats following insult with MCT (14). Accumulating evidence has indicated that growth factors, thrombin, angiotensin II, cytokines, transforming growth factor- $\beta$ or oxidative stress may account for HIF-1 $\alpha$ activation in PAH under non-hypoxic conditions (15). Of note, numerous of these aforementioned factors also stimulated the generation of reactive oxygen species (ROS), whereas inhibition of ROS formation decreased HIF-1 $\alpha$ protein levels, suggesting that ROS may have an important role in the regulation of HIF-1 $\alpha(16,17)$.

Therefore, it is hypothesized that DCA is effective in preventing or reversing intimal lesions by inhibition of ROS-induced HIF-1 $\alpha$ activation, which is mediated by upregulation or activation of SOD. In the present study, the effect of DCA on the pulmonary vascular remodeling in rats was examined, utilizing two regimens, consisting of a preventive or late intervention protocol. Furthermore, the HIF-1 $\alpha$ and ROS expression levels and SOD activity were detected and the possible mechanisms underlying DCA-induced amelioration of PAH were also investigated.

\section{Materials and methods}

Experimental animals. Healthy male Sprague-Dawley rats (nine weeks old) were purchased from Xipuer-Bikai Laboratory Animal Co., Ltd. (Shanghai, China) and housed in dry-raising cages ( $n=6 /$ cage) at the animal center of the Affiliated Hospital of Nanjing University Medical School (Nanjing, China) in a standard 12-h reverse day/night cycle at an ambient temperature of $26^{\circ} \mathrm{C}$. A diet of stock laboratory diet and tap water were available ad libitum. They were allowed a recovery period of one week prior to the experimental procedures. All experiments were performed with approval from the Committee of Nanjing University Medical School for Laboratory Animal Care and Use (Nanjing, China).

Left pneumonectomy. On day one, the rats were anesthetized with ketamine ( $150 \mathrm{mg} / \mathrm{kg}$, intraperitoneal injection) and orally intubated. Then, the rats underwent left pneumonectomy via left thoracotomy, as previously described (14).

MCT administration. MCT (Sigma-Aldrich, St. Louis, MO, USA) was dissolved in dimethyl sulfoxide (DMSO) at a concentration of $30 \mathrm{mg} / \mathrm{ml}$. On day eight, the rats were injected subcutaneously in the cervical areas with MCT $(2 \mathrm{ml} / \mathrm{kg})$ as previously described (14)

\section{Experimental groups}

Prevention study. Four groups of male Sprague-Dawley rats (weighing, 200-250 g; $n=8 /$ group) were used in the present study. Two groups of rats undergoing left unilateral pneumonectomized were injected with MCT $(60 \mathrm{mg} / \mathrm{kg}$, subcutaneous injection) seven days following surgery and the other two groups received sham surgery and DMSO injections. On day eight, one of the MCT- and DMSO-injected groups received $12 \mathrm{~g} / 1$ DCA (Sigma-Aldrich) gastric gavages at a dose of $80 \mathrm{mg} / \mathrm{kg}$ and the other two groups received the vehicle (normal saline) daily. On day 28, hemodynamic measurements were performed in the anesthetized rats.

Reversal study. The 32 male Sprague-Dawley rats were randomly divided into four groups as described above. On day 29, one of the MCT- and DMSO-injected groups received $12 \mathrm{~g} / \mathrm{l}$ DCA gastric gavages at a dose of $80 \mathrm{mg} / \mathrm{kg}$, whereas the other two groups received the vehicle (normal saline). Following 20 days of treatment, hemodynamic measurements and morphometric analyses were performed. The administration in the different groups is presented as a schematic in Fig. 1.

Hemodynamic studies and tissue preparation. At the end of the study as indicated above, the rats were anesthetized with ketamine (150 mg/kg, intraperitoneal injection). Their tracheas were orally intubated with a 16-gauge intravenous catheter and mechanical ventilation commenced using a rodent respirator (tidal volume, $8 \mathrm{ml} / \mathrm{kg}$; respiratory rate, $60 \mathrm{~min}^{-1}$ ). The rats' right carotid artery was cannulated with a 20-gauge intravenous catheter to record systemic blood pressure. Pulmonary hemodynamic parameters were measured in the open chest via direct puncture of the right ventricle outflow tract and then advancing the catheter into the pulmonary artery trunk (also confirmed by standard right ventricle or pulmonary arterial pressure trace on the monitor screen) connected to the pressure transducer of an Eagle400 monitor (General Electric Company, Fairfield, CT, USA). The right ventricle systolic pressure (RVSP), mean pulmonary arterial pressure (PAP) and mean carotid blood pressure (ABP) was recorded following 1 min of stabilization. All rats were then euthanized by exsanguination, and the right 


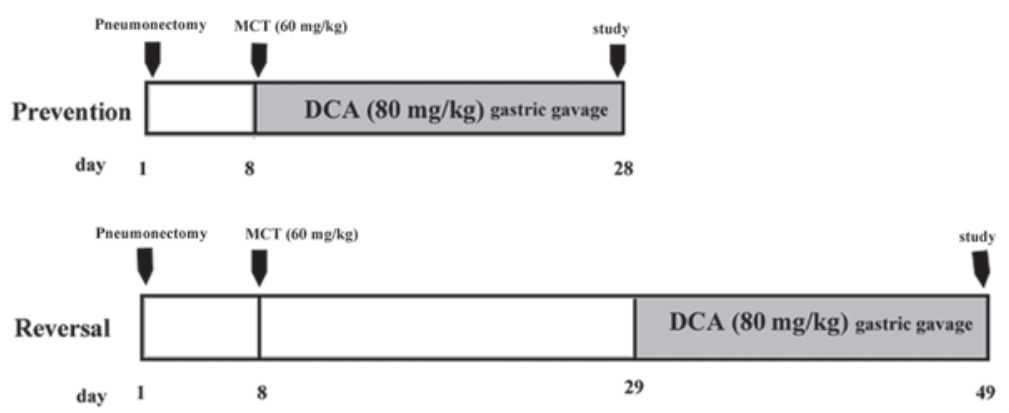

Figure 1. Schematic diagram depicting the experimental procedure. In the prevention group, the rats were subjected to $60 \mathrm{mg} / \mathrm{kg} \mathrm{MCT}$ cervical subcutaneous injection seven days following left pneumonectomy, and thereafter, the rats received DCA/vehicle $(80 \mathrm{mg} / \mathrm{kg})$ gastric gavage treatment per day from day 8-28. In the reversal group, the rats underwent left pneumonectomy and MCT cervical subcutaneous injection seven days following surgery. The rats received DCA $/$ vehicle $(80 \mathrm{mg} / \mathrm{kg})$ gastric gavage treatment per day from day 29-49. DCA, dichloroacetate; MCT, monocrotaline.

lungs (for histology and morphometry) and the hearts [for right ventricle (RV)-to-left ventricle (LV) + intra-ventricle septum (S) ratio $(\mathrm{RV} / \mathrm{LV}+\mathrm{IVS})]$ were collected. The right low lobe of lung was dissected, snap-frozen and maintained at a temperature of $-80^{\circ} \mathrm{C}$ until analyzed.

Morphological analysis. Histological changes of the pulmonary arteries (PAs) were quantified by morphometry as described below. Isolated lungs were inflated via the trachea with $10 \%$ formalin solution at $20 \mathrm{~cm} \mathrm{H}_{2} \mathrm{O}$ pressure and fixed in $10 \%$ formalin. Paraffin sections of $5 \mu \mathrm{m}$ were cut and stained with Elastin van Gieson and assessed microscopically to determine the degree of arterial wall thickness. In each lung section, ten small pulmonary arteries (PAs; 50-100 $\mu \mathrm{m}$ in diameter) were analyzed at a magnification of x400 in a blind manner. Medial wall thickness was expressed as the summation of two points of medial thickness/external diameter $\mathrm{x}$ 100 (\%). Intra-acinar (precapillary) PAs (25 vessels each) were assessed for occlusive lesions as grade 0 for no evidence of neointima lesion, grade 1 for $<50 \%$ luminal occlusion and grade 2 for $>50 \%$ luminal occlusion. There was no evidence of neointimal lesion formation in any PAs from normal rats (all PAs were graded as 0 ).

Immunohistochemistry. Slides were quenched in 3\% hydrogen peroxide and the antigen was retrieved using a pressure cooker in $10 \mathrm{mM}$ citrate buffer at $\mathrm{pH}$ 6. The slides were blocked in serum and the primary antibody was applied overnight at $4^{\circ} \mathrm{C}$. Immunodetection of HIF-1 $\alpha$ (dilution, 1:100; rabbit polyclonal; Novus Biologicals, Littleton, CO, USA) was performed with horseradish peroxidase-conjugated anti-rabbit secondary antibody (KPL, Inc., Gaithersburg, MD, USA) and DAB stain (Dako, Glostrup, Denmark). The slides were hematoxylin counterstained. The primary antibody (Dako Denmark A/S, Glostrup, Denmark) was omitted in the negative control for every group of slides.

Immunoblot assay. Whole cell, nuclear extracts were prepared according to manufacturer's instructions using the Nuclear Extract kit (Nanjing KeyGen Biotech. Co., Ltd., Nanjing, China). Whole cell extracts were prepared by lysing lung tissue in radioimmunoprecipitation assay buffer (protein extraction reagent with protease inhibitor cocktail, PMSF and phosphatase cocktail; Nanjing KeyGen Biotech., Co.,
Ltd., Nanjing, China) for $20 \mathrm{~min}$ on ice. Lysates were centrifuged $\left(4^{\circ} \mathrm{C}\right)$ at $16,000 \times \mathrm{g}$ for $10 \mathrm{~min}$ and the supernatant was collected for western blot analysis. Protein concentration was determined using the Bradford assay (Nanjing KeyGen Biotech. Co., Ltd.).

Western blot analysis. Western blots were performed by purifying $75 \mu \mathrm{g}$ of nuclear or whole cell protein extracts by $10 \%$ SDS-PAGE and transferring the purified protein to polyvinyl difluoride membranes by standard procedures. Membranes were blocked with tris-buffered saline/Tween-20 with $10 \%$ non-fat milk for $2 \mathrm{~h}$ and then either incubated overnight at $4^{\circ} \mathrm{C}$ or $4 \mathrm{~h}$ at room temperature with the respective primary antibody, including HIF-1 $\alpha$ (dilution, 1:500; Novus Biologicals), Kv1.5 (dilution, 1:1,000; Abcam, Cambridge, UK), survivin (1:1000 dilution; Abcam) and histone-3, GAPDH (each dilution, 1:2,000; Cell Signaling Technology, Inc., MA, USA). Immunoreactive bands were visualized using horseradish peroxidase-conjugated anti-mouse or anti-rabbit secondary antibody (dilution, 1:5,000; Upstate Biotechnology, Billerica, MA, USA) and enhanced chemiluminescence reagent (Western chemiluminescent detection system; Pierce Biotechnology, Inc., Rockford, IL, USA). Relative immunoreactive levels of proteins were quantified using the ChemiDoc XRS imaging system and Quantity One software (version 4.5; Bio-Rad, Hercules, CA, USA).

\section{Assessment of ROS and SOD}

Detection of ROS. For the tissue homogenates, lung tissue was rinsed with $1 \mathrm{X}$ phosphate-buffered saline (PBS) to remove excess blood, homogenized in $20 \mathrm{ml} 1 \mathrm{X}$ PBS and stored overnight at $<-20^{\circ} \mathrm{C}$. Following the two freeze-thaw cycles that were performed to break the cell membranes, the homogenates were centrifuged for $5 \mathrm{~min}$ at 5,000 $\mathrm{x} \mathrm{g}$. The supernatant was removed and assayed immediately or aliquoted and stored at $<-20^{\circ} \mathrm{C}$. ROS was detected according to the manufacturer's instructions by using a rat ROS ELISA assay kit (R\&D Systems, Inc., Minneapolis, MN, USA).

SOD activity assay. Prior to the assays, tissue samples were thawed on ice and homogenized (Polytron PT3100; Brinkman Instruments, Littau, Switzerland) in selected buffers. The total superoxide dismutase activities were measured by using a water-soluble formazan dye kit (Dojindo Molecular Technologies, Inc., Shanghai, China) according to 
A

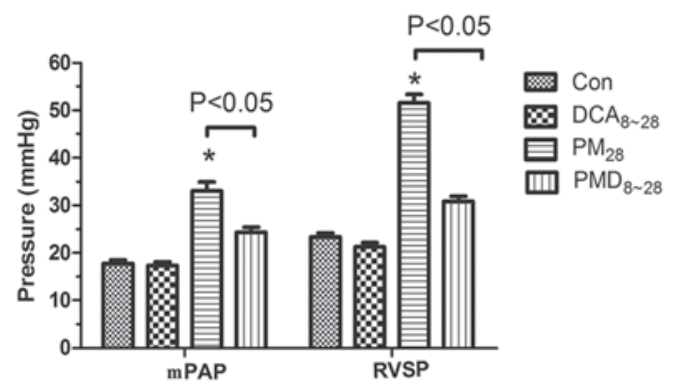

B

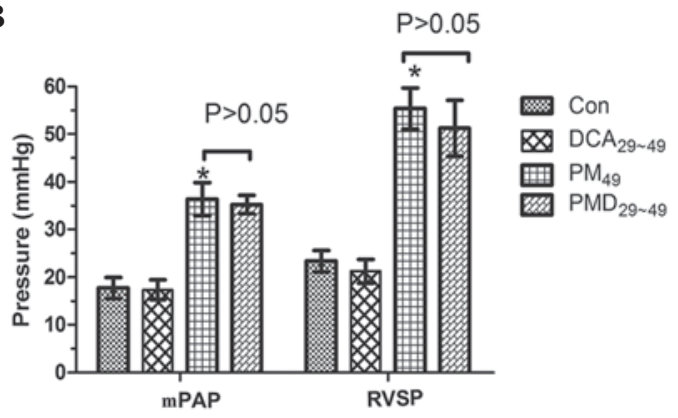

Figure 2. Effect of DCA on the hemodynamic parameters in MCT-treated pneumonectomized rats. The MCT-treated pneumonectomized rats received preventive (day 8-28) and delayed (day 29-49) DCA gastric gavage treatment, respectively. At the end of the study, pulmonary hemodynamic parameters (mPAP and RVSP) were measured on (A) day 28 and (B) day 49, respectively. The data are presented as the mean \pm standard error of the mean $(n=8)$. "P<0.05 vs. reference control rats on day 28 or 49. Con, rats subjected to sham surgery and DMSO and treated with vehicle (saline); DCA, rats subjected to sham surgery and DMSO and treated with DCA ( $80 \mathrm{mg} / \mathrm{kg}$ daily) gavage treatment; $\mathrm{PM}_{28}$, rats subjected to pneumonectomy and MCT and treated with vehicle from day $8-28 ; \mathrm{PMD}_{8 \sim 28}$ rats subjected to pneumonectomy and MCT and treated with DCA from day 8-28; $\mathrm{PM}_{49}$, rats subjected to pneumonectomy and MCT and treated with vehicle from day 29-49; $\mathrm{PMD}_{29 \sim 49}$, rats subjected to pneumonectomy and MCT and treated with DCA from day 29-49. mPAP, mean pulmonary arterial pressure; DCA, dichloroacetate; MCT, monocrotaline; RVSP, right ventricular systolic pressure; DMSO, dimethyl sulfoxide.

the manufacturer's instructions. To measure manganese SOD (MnSOD) activity, copper/zinc SOD (Cu/Zn-SOD) activity was blocked with $1 \mathrm{mmol} / \mathrm{l}$ potassium cyanide. The amount of protein was measured using a bicinchoninic acid protein assay (Sigma-Aldrich). Enzymatic activity was expressed in units/mg protein (U/mgprot).

Statistical analysis. Statistical analysis was performed with GraphPad Prism 5 (GraphPad Software, San Diego, CA, USA). The results are presented as the mean \pm standard error of the mean. Analysis of variance followed by Bonferroni's post-hoc test to compare the mean values among the various groups. A P-value of $<0.05$ was considered to indicate a statistically significant difference.

\section{Results}

Effect of DCA on hemodynamic parameters and the formation of neointimal lesions. Consistent with previous results, left unilateral pneumonectomy plus MCT treatment caused a significant increase in the mean PAP, RVSP and right ventricle hypertrophy in rats as compared with the reference control rats on day 28 (mean PAP, $33 \pm 5.5$ vs. $18 \pm 2.1 \mathrm{mmHg}$; RVSP, $52 \pm 5.3$ vs. $23 \pm 2.2 \mathrm{mmHg}$; RV/LV+IVS, $43 \pm 3.3$ vs. $28 \pm 2.2 \%$; $\mathrm{P}<0.05$ ). The preventive treatment with DCA significantly repressed the elevation of pulmonary pressure (mean PAP, $24 \pm 2.8$ vs. $33 \pm 5.5 \mathrm{mmHg}$; RVSP, $31 \pm 3.2$ vs. $52 \pm 5.3 \mathrm{mmHg}$; $\mathrm{P}<0.05)$ and right ventricle hypertrophy (RV/LV+IVS, $29 \pm 2.8$ vs. $43 \pm 3.3 \%$ ) in pulmonary hypertensive rats (Figs. $2 \mathrm{~A}$ and $3 \mathrm{H}$ ).

Of note, the MCT-treated pneumonectomized rats exhibited severe vascular remodeling, including medial wall hypertrophy and occlusive neointimal formation leading to partial, even complete, obliteration of pulmonary arterioles on day 28 (Figs. 3B and 4A). In the pulmonary arteries (50 100 $\mu \mathrm{m}$ in diameter), the percentage of medial wall thickness was markedly increased following left pneumonectomy plus MCT insults from $15 \%$ in the reference control rats to $78 \%$, which was reduced by the treatment with early intervention of DCA $(51 \pm 8.1$ vs. $78 \pm 12.0 \%$; $\mathrm{P}<0.05$; Fig. $3 \mathrm{G})$. Furthermore, Fig. 4B and $\mathrm{E}$ reveal that the percentage of grade 1 and 2 occlusion in pre-acinar arteries accounted for 24 and $20 \%$ of all pulmonary arterioles measured, respectively, following treatment with DCA, which represented a marked alleviation in neointimal lesion formation as compared with the MCT-treated pneumonectomized rats receiving normal saline from 8-28 days following pneumonectomy (grade 1 and 2 occlusion accounting for 44 and $40 \%$, respectively).

Compared with the MCT-treated pneumonectomized rats on day 49, intervention with DCA from day 29-49 had little effect on PAH (mean PAP, $35 \pm 1.9$ vs. $36 \pm 3.5 \mathrm{mmHg}$; RVSP, $51 \pm 5.9$ vs. $55 \pm 4.3 \mathrm{mmHg}$; P $>0.05$; Fig. $2 \mathrm{~B}$ ), RV hypertrophy (RV/LV+IVS, $55 \pm 1.9 \%$ vs. $58 \pm 3.8 \%$; P $>0.05$; Fig. $3 \mathrm{H})$ and vascular remodeling (medial wall thickness, $87 \pm 6.0$ vs. $86 \pm 8.1 \%$; grade 1 and 2 occlusion, 32 and 64 vs. $32 \%$ and $60 \%$; P>0.05; Fig. 3G, 4C-E).

Effect of DCA on HIF-1 1 expression, voltage-dependent potassium channel subtype $1.5(\mathrm{Kv} 1.5)$ and survivin expression. Fig. 5B and D show intense staining of HIF-1 $\alpha$ in occlusive neointimal lesions in MCT-treated pneumonectomized rats on day 28 and 49, respectively. The immunohistochemical and western blotting assays indicated that the early treatment with DCA significantly inhibited HIF-1 $\alpha$ activation, as illustrated in Fig. 5C and Fig. 6A and B. By contrast, the activation of HIF-1 $\alpha$ was not inhibited by DCA treatment from day 29-49 (Fig. 5E, and Fig. 6A and B).

The findings from the present study demonstrated that the reduced expression of Kv1.5 was restored by early intervention with DCA in MCT-treated pneumonectomized rats. However, when DCA treatment was delayed, there was evident restoration of Kv1.5 expression on day 49 in rats (Fig. 6C and D).

There is considerable evidence that survivin is a key anti-apoptotic factor responsible for cell overgrowth (18). In the present study, the expression of survivin in the MCT-treated pneumonectomized rats was $\sim 4$-fold higher as compared with the normal control rats, which was abrogated with DCA treatment $(\mathrm{P}<0.05)$. As demonstrated in Fig. 6E and $\mathrm{F}$, however, the expression of survivin was not significantly different between the MCT-treated pneumonectomized rats receiving vehicle or DCA from day 29-49. 

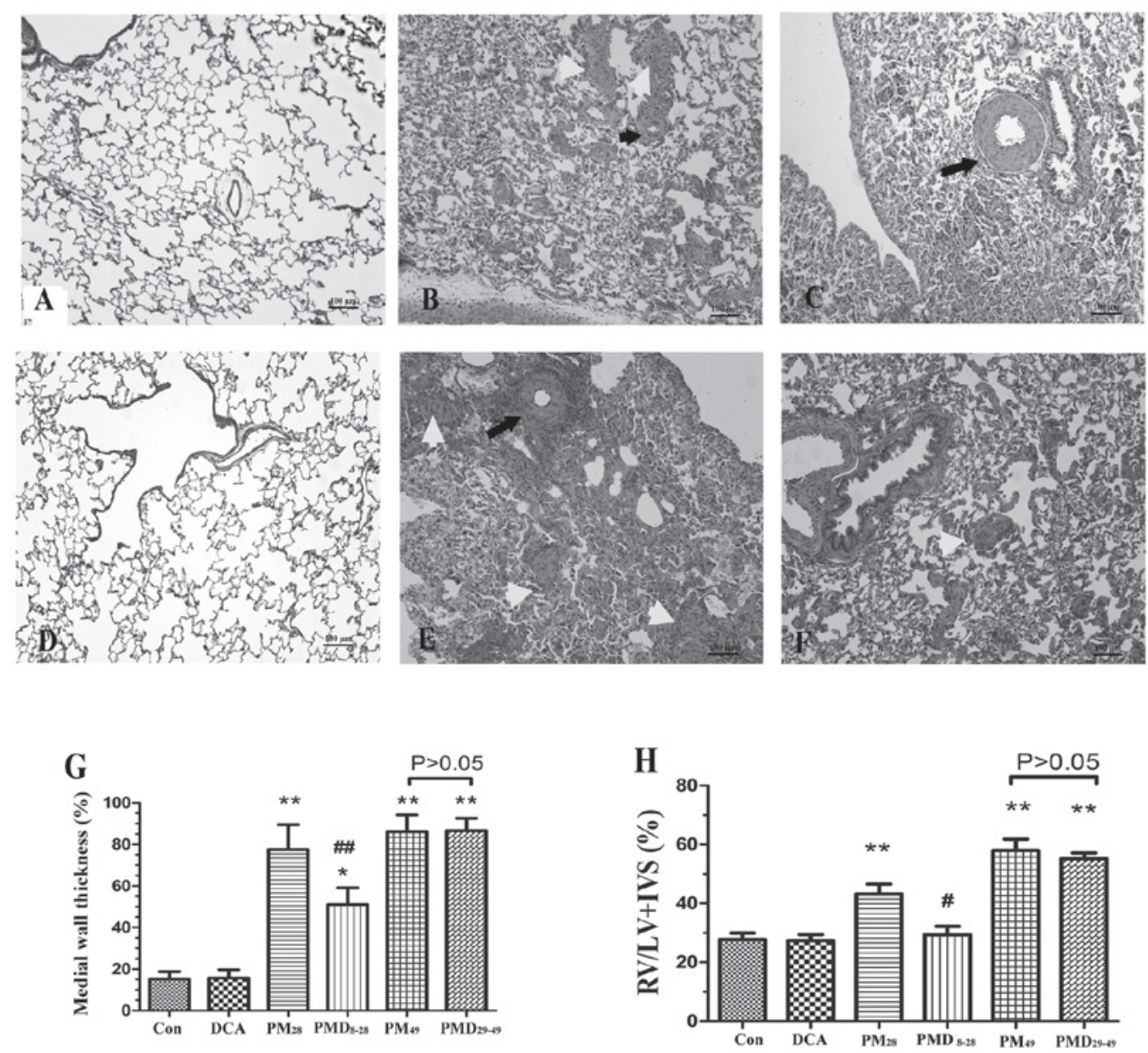

Figure 3. Effect of DCA on pulmonary arterial medial wall thickness and RV/LV+IVS. The MCT-treated pneumonectomized rats received preventive (day 8-28) and delayed (day 29-49) DCA gastric gavage treatment, respectively. At the end of the study, the right lung and heart were dissected for determination of lung vascular injury and right ventricle hypertrophy, respectively. Rat lung tissues were stained with H\&E. View of lung (magnification, $\mathrm{x} 10)$ from a rat subjected to sham operation and DMSO and treated with (A) saline or (D) DCA receiving vehicle from day 8-28. Rat subjected to pneumonectomy and treated with MCT receiving (B) vehicle or (C) DCA gastric gavage from day 8-28. Rat subjected to pneumonectomy and treated with MCT receiving (E) vehicle or (F) DCA gastric gavage from day 29-49. The vessels marked with black arrows exhibited medial hypertrophy and luminal narrowing and white arrow heads indicating completed obliteration of several small arteriole lumens. (H\&E stain; scale bar in A-F, $100 \mu \mathrm{m}$ ). (G) Effect of DCA treatment on the percentage of medial thickness in pulmonary arterioles and $(\mathrm{H})$ right ventricle hypertrophy index. The data are presented as the mean \pm standard error of the mean $(\mathrm{n}=8)$. ${ }^{*} \mathrm{P}<0.05,{ }^{* *} \mathrm{P}<0.01$ vs. reference control rats. ${ }^{\#} \mathrm{P}<0.05,{ }^{\# /} \mathrm{P}<0.01$ vs. MCT-treated pneumonectomized rats receiving vehicle from day $8-28$. Con, rats subjected to sham surgery and DMSO and treated with vehicle (saline); DCA, rats subjected to sham surgery and DMSO and treated with DCA (80 mg/kg daily) gavage treatment; $\mathrm{PM}_{28}$, rats subjected to pneumonectomy and $\mathrm{MCT}$ and treated with vehicle from day $8-28 ; \mathrm{PMD}_{8 \sim 28}$, rats subjected to pneumonectomy and MCT treated with DCA from day 8-28; $\mathrm{PM}_{49}$, rats subjected to pneumonectomy and MCT and treated with vehicle from day $29-49 ; \mathrm{PMD}_{29 \sim 49}$, rats subjected to pneumonectomy and MCT and treated with DCA from day 29-49. DMSO, dimethyl sulfoxide; DCA, dichloroacetate; MCT, monocrotaline; RV/LV+IVS, right ventricle/left ventricle+ intra-ventricle septum; H\&E, hematoxylin and eosin.

Effect of DCA on ROS content and the expression of SOD in lung tissue. In agreement with the previous studies, the rats that underwent pneumonectomy and treatment with MCT exhibited higher ROS production compared with the rats subjected to sham surgery plus DMSO treatment $(106 \pm 4.7$ vs. $56 \pm 10.2 \mathrm{U} / \mathrm{mgprot} ; \mathrm{P}<0.05$ ) on day 28 . As demonstrated in Fig. 7A, the intervention with DCA effectively suppressed the increase of ROS, which was reduced by almost $25 \%$ as compared with the MCT-treated pneumonectomized rats receiving vehicle on day 28 . However, the MCT-treated pneumonectomized rats treated with DCA from day 29-49 had a higher ROS content as compared with those receiving vehicle treatment $(115 \pm 8.8$ vs. $98 \pm 5.4 \mathrm{U} / \mathrm{mgprot}$; $\mathrm{P}>0.05)$.

As is well-established, SOD has a key role in the degradation of ROS. The activity of total SOD, MnSOD and Cu/Zn SOD was investigated in the present study. The total SOD activity was significantly suppressed in MCT-treated pneumonectomized rats, which was, in part, restored by early intervention with DCA $(66 \pm 4.4$ vs. $106 \pm 21.8 \mathrm{U} / \mathrm{mgprot}$; $\mathrm{P}<0.05$; Fig. 7B). The activity of MnSOD did not exhibit any significant difference among the six groups, as illustrated in Fig. 7C. However, of note, the activity changes of total SOD were in parallel with the alteration of $\mathrm{Cu} / \mathrm{Zn}$ SOD activity. Fig. 7D reveals that the activity of $\mathrm{Cu} / \mathrm{Zn}$ SOD was markedly suppressed in MCT-treated pneumonectomized rats on day 28 as compared with that in the normal control rats $(53 \pm 2.7$ vs. $96 \pm 19.5 \mathrm{U} / \mathrm{mgprot} ; \mathrm{P}<0.05)$, which was reversed by intervention with DCA $(88 \pm 4.2$ vs. $53 \pm 2.7 \mathrm{U} / \mathrm{mgprot}$; $\mathrm{P}<0.05)$. However, late intervention with DCA had a weak effect on $\mathrm{Cu} / \mathrm{Zn}$ SOD activity (Fig. 7D). Of note, the MCT-treated pneumonectomized rats on day 49 exhibited an unexpected increase of $\mathrm{Cu} / \mathrm{Zn}$ SOD activity as compared with the 

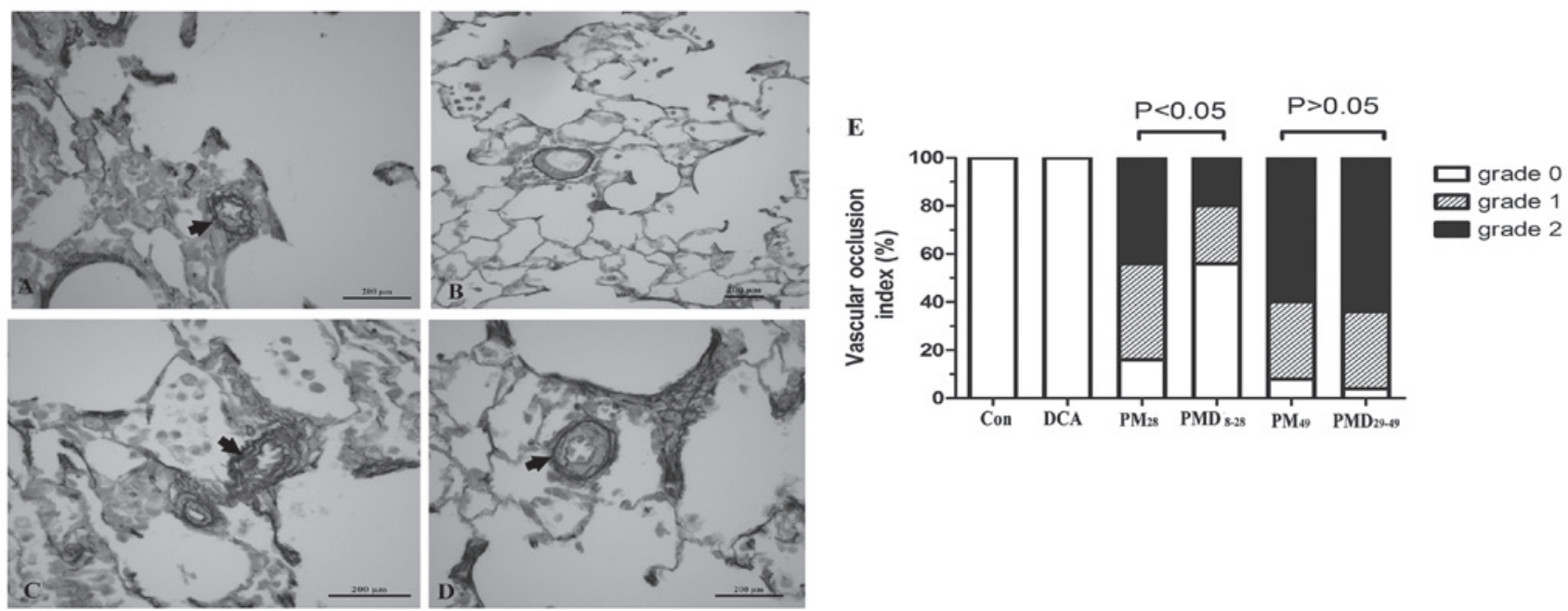

Figure 4. Effect of DCA on pulmonary arterioles intimal lesion formation in rats treated with MCT following pneumonectomy. The MCT-treated pneumonectomized rats received preventive (day 8-28) and delayed (day 29-49) DCA gastric gavage treatment, respectively. At the end of the experimental procedures, the rat lung tissues were stained with Elastin van Gieson to delineate the intima and media in pulmonary arterioles. View of lung (magnification, $\mathrm{x} 40$ ) from a rat subjected to pneumonectomy and treated with MCT receiving (A) vehicle or (B) DCA gastric gavage from day 8-28. Rats subjected to pneumonectomy and treated with MCT receiving (C) vehicle or (D) DCA gastric gavage from day 29-49. The vessels marked with arrows exhibited internal elastic laminal interruption and neointimal formation, causing the narrowing of pulmonary arteriole lumens. Scale bar in A-D, $200 \mu \mathrm{m}$. (E) Changes of the vascular occlusion index of pulmonary arterioles following preventive or delayed DCA treatment. Con, rats subjected to sham surgery and DMSO and treated with vehicle (saline); DCA, rats subjected to sham surgery and DMSO and treated with DCA; $\mathrm{PM}_{28}$, rats subjected to pneumonectomy and MCT and treated with vehicle from day 8-28; $\mathrm{PMD}_{8 \sim 28}$, rats subjected to pneumonectomy and MCT and treated with DCA from day 8-28; $\mathrm{PM}_{49}$, rats subjected to pneumonectomy and MCT and treated with vehicle from day 29-49; $\mathrm{PMD}_{29 \sim 49}$, rats subjected to pneumonectomy and MCT and treated with DCA from day 29-49; DCA, dichloroacetate; MCT, monocrotaline; DMSO, dimethyl sulfoxide.
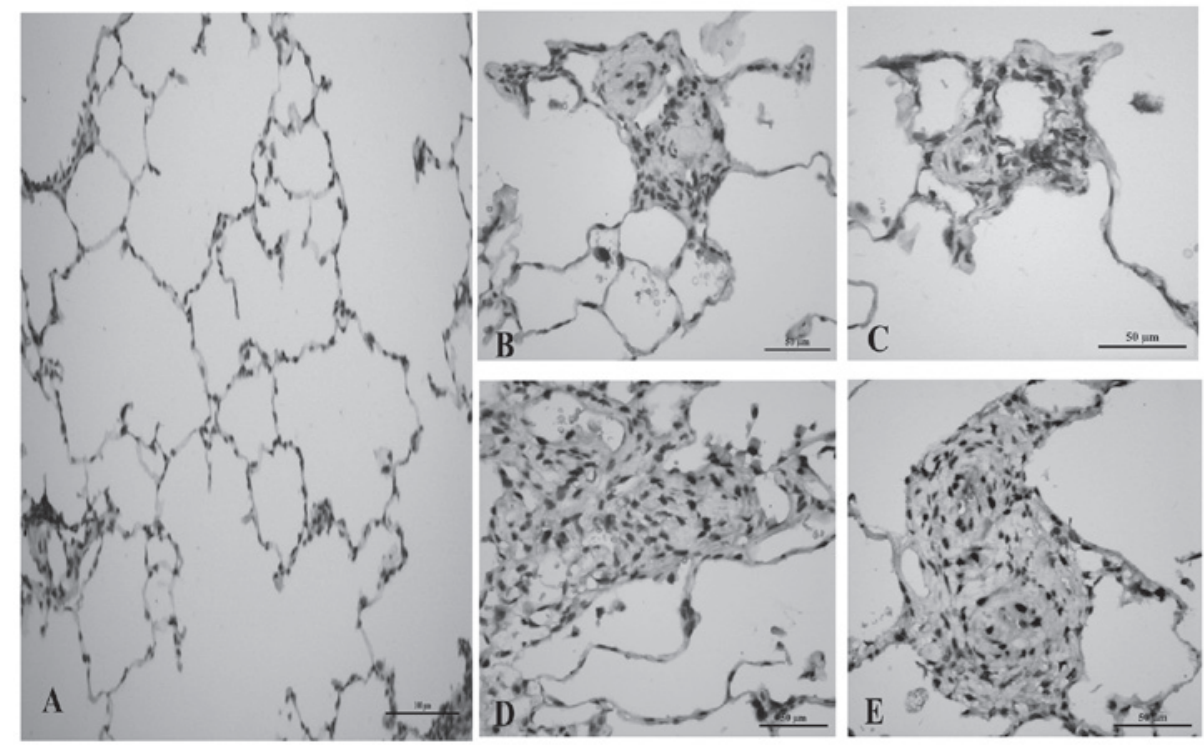

Figure 5. Immunohistochemical staining of HIF-1 $\alpha$ in pulmonary arterioles. Lung tissue from (A) Con, (B) $\mathrm{PM}_{28}$, (C) $\mathrm{PMD}_{8 \sim 28}$, (D) $\mathrm{PM}_{49}$ and (E) PMD $29 \sim 49$. (magnification in A, x10; magnification in B-E, x40; scale bar in A, $100 \mu \mathrm{m}$; scale bar in B-E, $50 \mu \mathrm{m}$ ). Con, rats subjected to sham surgery and DMSO and treated with vehicle (saline); $\mathrm{PM}_{28}$, rats subjected to pneumonectomy and $\mathrm{MCT}$ and treated with vehicle from day $8-28$; $\mathrm{PMD}_{8 \sim 28}$, rats subjected to pneumonectomy and MCT and treated with DCA from day 8-28; $\mathrm{PM}_{49}$, rats subjected to pneumonectomy and MCT and treated with vehicle from day 29-49; $\mathrm{PMD}_{29 \sim 49}$, rats subjected to pneumonectomy and MCT and treated with DCA from day 29-49. HIF-1 $\alpha$, hypoxia-inducible factor-1 $\alpha$; DCA, dichloroacetate; MCT, monocrotaline; DMSO, dimethyl sulfoxide.

MCT-treated pneumonectomized rats receiving the vehicle from day $8-28$.

\section{Discussion}

The results of the present study indicated that DCA was effective in preventing the formation of neointimal lesions in pulmonary arterioles of MCT-induced pneumonectomized rats and increases in PAP. This effect may be attributed to $\mathrm{Cu} / \mathrm{Zn}$ SOD upregulation, HIF-1 $\alpha$ inactivation and subsequent cell apoptosis in the neointimal lesion areas. By contrast, late intervention with DCA demonstrated little efficacy in the amelioration of PAP and severe structural destruction in pulmonary arterioles. 
A

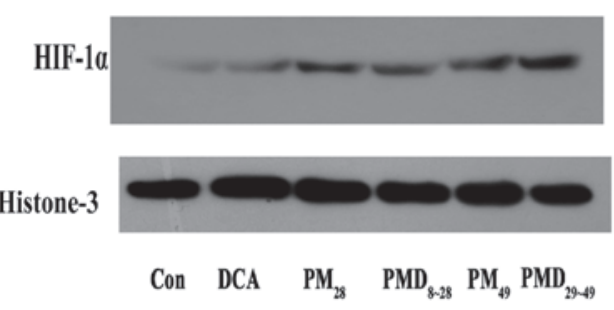

C
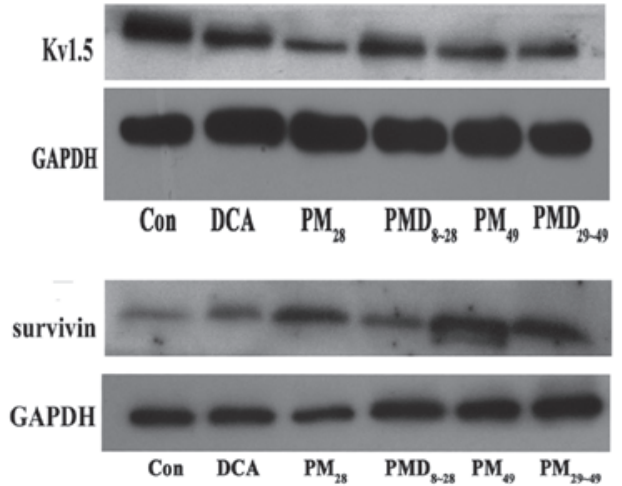

B

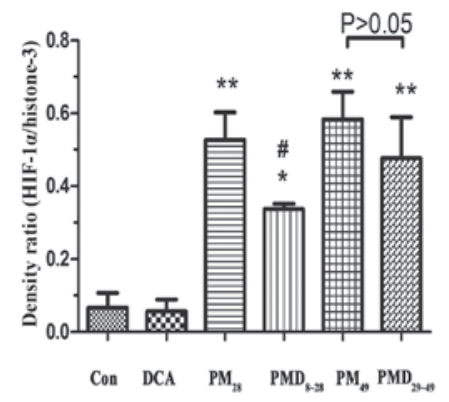

D
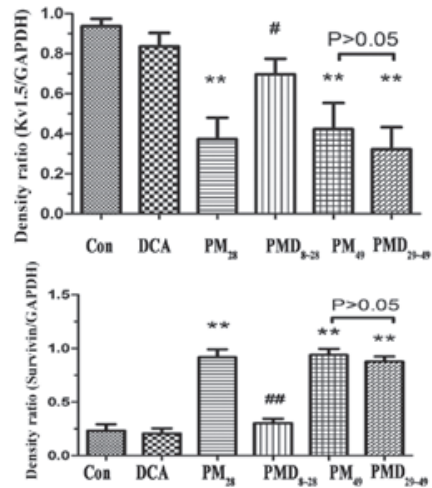

Figure 6. Western blot analysis of expression of HIF-1 $\alpha$, Kv1.5 and survivin in lung tissue. (A) Representative alteration of HIF-1 $\alpha$ expression in one of three independent experiments with identical results. (B) Density ratio of HIF-1 $\alpha$ to Histone-3. (C) Representative alteration of Kv1.5 or survivin, expression in one of three separate experiments with the identical results. (D) Density ratio of Kv1.5 or survivin to GAPDH. The data are presented as the mean \pm standard error of the mean ( $\mathrm{n}=3) .{ }^{*} \mathrm{P}<0.05,{ }^{* *} \mathrm{P}<0.01$ vs. Con; ${ }^{*} \mathrm{P}<0.05$ vs. $\mathrm{PM}_{28}$. Con, rats subjected to sham surgery and DMSO and treated with vehicle (saline); DCA, rats subjected to sham surgery and DMSO and treated with DCA $\left(80 \mathrm{mg} / \mathrm{kg}\right.$ daily) gavage treatment; $\mathrm{PM}_{28}$, rats subjected to pneumonectomy and MCT and treated with vehicle from day 8-28; $\mathrm{PMD}_{8 \sim 28}$, rats subjected to pneumonectomy and MCT treated with DCA from day 8-28; $\mathrm{PM}_{49}$, rats subjected to pneumonectomy and MCT and treated with vehicle from day $29-49 ; \mathrm{PMD}_{29 \sim 49}$, rats subjected to pneumonectomy and MCT and treated with DCA from day 29-49. Kv1.5, voltage-dependent potassium channel subtype 1.5; HIF-1 $\alpha$, HIF-1 $\alpha$, hypoxia-inducible factor-1 $\alpha$; MCT, monocrotaline; DCA, dichloroacetate; DMSO, dimethyl sulfoxide.

A

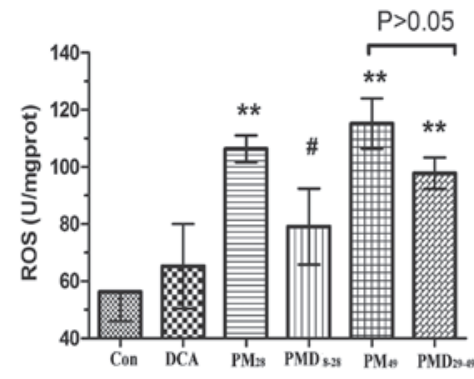

C

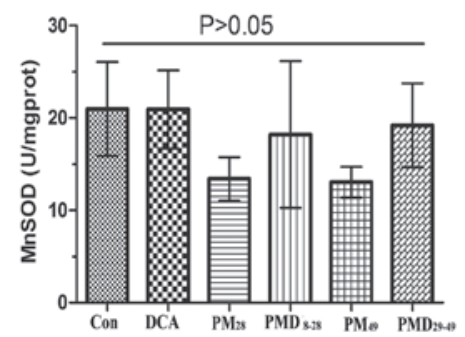

B

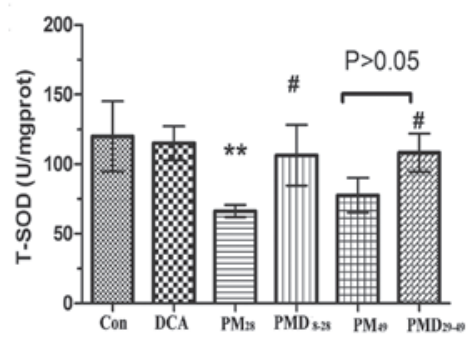

D

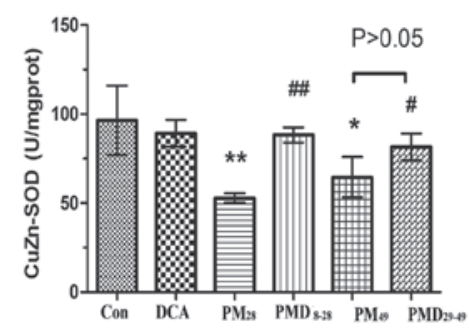

Figure 7. Effect of DCA on ROS production and SOD activation. The MCT-treated pneumonectomized rats received early (day 8-28) and delayed (day 29-49) DCA gastric gavage treatment, respectively. At the end of the study, the content of (A) ROS, (B) total SOD, (C) MnSOD, (D) Cu/Zn SOD in rat lung tissue was measured by a colorimetric assay. Data are presented as the mean \pm standard error of the mean $(\mathrm{n}=8)$. ${ }^{*} \mathrm{P}<0.05$, ${ }^{* *} \mathrm{P}<0.01 \mathrm{vs}$. Con; ${ }^{*} \mathrm{P}<0.05$ vs. $\mathrm{PM}_{28}$. Con, rats subjected to sham surgery and DMSO and treated with vehicle (saline); DCA, rats subjected to sham surgery and DMSO and treated with DCA $\left(80 \mathrm{mg} / \mathrm{kg}\right.$ daily) gavage treatment; $\mathrm{PM}_{28}$, rats subjected to pneumonectomy and MCT and treated with vehicle from day $8-28 ; \mathrm{PMD}_{8 \sim 28}$, rats subjected to pneumonectomy and MCT and treated with DCA from day 8-28; $\mathrm{PM}_{49}$, rats subjected to pneumonectomy and MCT and treated with vehicle from day 29-49; $\mathrm{PMD}_{29 \sim 49}$, rats subjected to pneumonectomy and MCT and treated with DCA from day 29-49. ROS, reactive oxygen species; SOD, superoxide dismutase; MCT, monocrotaline; DCA, dichloroacetate; MnSOD, manganese SOD; Cu/Zn SOD, copper/zinc SOD; DMSO, dimethyl sulfoxide. 
DCA has been widely employed in a number of diseases associated with mitochondrial dysfunction for approximately three decades. Recent studies have demonstrated the beneficial effect of DCA in MCT or hypoxic PAH models $(5,6)$. However, these aforementioned rat models are limited by their inability to reproduce the full spectrum of vascular pathological injuries observed in lung specimens from PAH patients. In particular, these models lack the characteristic neointimal formation which causes narrowing, or even obliteration of vessel lumens in pre-capillary vessels, as well as the significant elevation in pulmonary vascular resistance (19). Intimal lesions are commonly regarded as a type of pulmonary vascular pathology representing severe injury and represent the irreversibility status during pulmonary vascular remodeling (20). Therefore, it is highly important to prevent or reverse the formation of neointimal lesions. The rats undergoing left lung resection followed by MCT subcutaneous injection developed severe $\mathrm{PAH}$, accompanied with severe occlusive neointimal formation in pulmonary arterioles. Since its first introduction by Okada et al (21), this model has been applied to test the effectiveness of a variety of drugs (simvastatin, triptolide, etc.) in PAH (21-23). However, the efficacy of DCA in this rat model, to the best of our knowledge, has not been investigated to date.

DCA is a well-characterized inhibitor of the protein kinase of pyruvate dehydrogenase (PDH). DCA-mediated inhibition of PDH kinase renders the majority of $\mathrm{PDH}$ in the active form, which triggers a switch in pyruvate metabolism towards glucose oxidation to $\mathrm{CO}_{2}$ in the mitochondria (24). Early intervention with DCA in the present study effectively attenuated neointimal formation in pulmonary hypertensive rats. This effect was accompanied by the suppression of HIF- $1 \alpha$ activation and the induction of Kv1.5 expression in the neointimal lesions. By contrast, no amelioration in pulmonary vascular remodeling was observed in the late intervention group. Concomitantly, late treatment with DCA did not reverse the alterations of this transcription factor and Kv1.5 protein expression in pulmonary hypertensive rats. These data suggested that HIF-1 $\alpha$ inactivation and upregulation of Kv1.5 may be the key factors responsible for preventing neointimal formation in MCT-induced pneumonectomized rats treated with DCA. Accumulating evidence indicated that MnSOD (SOD2) converts intramitochondrial superoxide to diffusible hydrogen peroxide, which serves as a redox-signaling molecule, regulating pulmonary vascular tone and structure through effects on Kv1.5 and transcription factors (15). The expression of MnSOD has been identified as reduced in idiopathic PAH patients and in fawn-hooded rats that spontaneously develop PAH $(25,26)$. The reduced expression or inactivation of MnSOD creates a pseudohypoxic redox state in PAH characterized by normoxic decreases in ROS, a shift from oxidative to glycolytic metabolism and HIF-1 $\alpha$ activation (27). DCA corrects the mitochondrial abnormalities in several experimental models of $\mathrm{PAH}$, causing a regression of remodeled pulmonary vasculature. Inconsistent with previous results, in the present study, HIF-1 $\alpha$ inactivation was attributed to DCA-induced elevation in the activity of $\mathrm{Cu} / \mathrm{Zn}$ SOD (SOD1 and SOD3), while changes in MnSOD levels were not statistically significant $(\mathrm{P}>0.05)$. Experimental findings from Dorfmüller et al (28) indicated that increased oxidative stress and severe vascular remodeling occurred in MCT-treated rats following left pneumonectomy. In addition, administration of antioxidants attenuated the development of PAH, which suggested that pulmonary oxidative stress regulates the development of PAH (29). In the present study, a change with opposite trends was observed between ROS and total SOD production in rat lung specimens 20 days following MCT treatment. Early treatment with DCA effectively prevented excessive ROS formation, which was mediated by an upregulation of $\mathrm{Cu} / \mathrm{Zn}$ SOD activity. SOD catalyzes the dismutation of superoxide into oxygen and hydrogen peroxide, and elevated hydrogen peroxide and NO levels are known to block the induction of HIF-1 $\alpha$-associated genes and the accumulation of HIF-1 $\alpha$ (30). Therefore, it is hypothesized that DCA-induced elevation of $\mathrm{Cu} / \mathrm{Zn}$ SOD activity may be involved in the inactivation of HIF-1 $\alpha$ in neointimal lesion areas. In accordance with the results of the present study, the findings from Saed et al (11) indicated that SOD3 was upregulated in a dose-dependent manner at the transcriptional and protein levels following DCA treatment, which induced apoptosis, reduced myeloperoxidase activation and HIF-1 $\alpha$ transcription in two epithelial ovarian cancer cell lines. The protective effect of SOD3 has also been confirmed by a study demonstrating that the overexpression of SOD3 in bleomycin induced pulmonary hypertension in rats (31). By contrast, in another study, enhanced hydrogen peroxide levels promoted thrombin-induced HIF-1 $\alpha$ upregulation, whereas degradation of hydrogen peroxide with catalase prevented HIF-1 $\alpha$ activation in pulmonary arterial SMCs (32). Therefore, the regulatory role of hydrogen peroxide in the induction of HIF-1 $\alpha$ activation remains elusive. A study by Görlach et al (33) indicated that higher concentrations of hydrogen peroxide were more inclined to prevent HIF-1 $\alpha$ accumulation, suggesting that only moderate changes in the redox state are required to activate the HIF pathway. By contrast, a substantial increase in hydrogen peroxide levels (as they may be observed at conditions of oxidative stress) does inactivate the HIF system. In addition, the present study did not observe any significant changes in the activity and protein expression of MnSOD (data not shown). The rodent model used in the present study, which is characterized by enhanced oxidative stress and inflammatory cell recruitment in lungs, may account for the distinct roles of three types of SOD in HIF-1 $\alpha$ suppression.

There are several possible explanations for the late intervention with DCA not being effective in the PAH rats. Firstly, 20 days following MCT insults in pneumonectomized rats, the occlusion of pulmonary arterioles was severe, which compromised the trafficking and absorption of DCA at the sites of neointimal lesions. Secondly, as is consistent with the results by Dorfmüller et al (28), ROS in the lung tissue were markedly increased following MCT treatment in pneumonectomized rats. The early release of ROS and the occurrence of damage to the adjacent tissues may have produced enough ROS to injure their distant target (pulmonary arteries) and trigger a self-enhancing pro-oxidative and pro-inflammatory process, which may not be affected by any delayed ROS-dependent therapy (34).

In conclusion, the present study showed that HIF-1 $\alpha$ was upregulated in the neointimal lesion areas of pulmonary arterioles in MCT-treated pneumonectomized rats, which accounts for the occlusive neointimal formation and severe 
pulmonary hypertension. The results demonstrated that DCA was effective in the upregulation of $\mathrm{Cu} / \mathrm{Zn}$ SOD activation leading to HIF-1 $\alpha$ inactivation in pulmonary vessels. However, late treatment with DCA was not able to attenuate pulmonary vascular remodeling and hemodynamics in this well established PAH model. Investigating methods to improve DCA transportation to neointimal lesion areas and inhibit the amplified oxidative stress may be a novel strategy for the treatment of PAH in the future.

\section{Acknowledgements}

This study was supported by grants from National Natural Science Foundation Committee of China (project no.30901406); Young Health Talents Project from Nanjing Municipal Health Bureau; Natural Science Foundation Committee of Jiangsu Province (BK2012532) and Fundamental Research Fund for Central University of Nanjing University, China.

\section{References}

1. McLaughlin VV, Davis M and Cornwell W: Pulmonary arterial hypertension. Curr Probl Cardiol 36: 461-517, 2011.

2. Tuder RM: Pathology of pulmonary arterial hypertension. Semin Respir Crit Care Med 30: 376-385, 2009.

3. Stamm JA, Risbano MG and Mathier MA: Overview of current therapeutic approaches for pulmonary hypertension. Pulm Circ 1: 138-159, 2011.

4. Stacpoole PW, Nagaraja NV and Hutson AD: Efficacy of dichloroacetate as a lactate-lowering drug. J Clin Pharmacol 43: 683-691, 2003.

5. McMurtry MS, Bonnet S, Wu X, Dyck JR, Haromy A, Hashimoto $\mathrm{K}$, et al: Dichloroacetate prevents and reverses pulmonary hypertension by inducing pulmonary artery smooth muscle cell apoptosis. Circ Res 95: 830-840, 2004.

6. Michelakis ED, McMurtry MS, Wu XC, Dyck JR, Moudgil R, Hopkins TA, et al: Dichloroacetate, a metabolic modulator, prevents and reverses chronic hypoxic pulmonary hypertension in rats: role of increased expression and activity of voltage-gated potassium channels. Circulation 105: 244-250, 2002 .

7. Guignabert C, Tu L, Izikki M, Dewachter L, Zadigue P, Humbert M, et al: Dichloroacetate treatment partially regresses established pulmonary hypertension in mice with SM22alpha-targeted overexpression of the serotonin transporter. FASEB J 23: 4135-4147, 2009.

8. Michelakis ED, Webster L and Mackey JR: Dichloroacetate (DCA) as a potential metabolic-targeting therapy for cancer. Br J Cancer 99: 989-994, 2008.

9. Wong JY, Huggins GS, Debidda M, Munshi NC and De Vivo I: Dichloroacetate induces apoptosis in endometrial cancer cells. Gynecol Oncol 109: 394-402, 2008.

10. Washington JT and Quintyne NJ: Dichloroacetate induces different rates of cell death in cancer and noncancer cell lines in vitro. Tumori 98: 142-151, 2012.

11. Saed GM, Fletcher NM, Jiang ZL, Abu-Soud HM and Diamond MP: Dichloroacetate induces apoptosis of epithelial ovarian cancer cells through a mechanism involving modulation of oxidative stress. Reprod Sci 18: 1253-1261, 2011.

12. Semenza GL: Involvement of hypoxia-inducible factor 1 in pulmonary pathophysiology. Chest 128 (Suppl): 592S-594S, 2005.

13. Tuder RM, Chacon M, Alger L, Wang J, TarasevicieneStewartL,Kasahara Y,et al:Expression of angiogenesis-related molecules in plexiform lesions in severe pulmonary hypertension: evidence for a process of disordered angiogenesis. J Pathol 195: 367-374, 2001

14. Yan J, Shen Y, Wang Y and Li BB: Increased expression of hypoxia inducible factor- $1 \alpha$ in proliferating neointimal lesions in a rat model of pulmonary arterial hypertension. Am J Med Sci 345: 121-128, 2013.
15. Archer SL, Gomberg-Maitland M, Maitland ML, Rich S, Garcia JG and Weir EK: Mitochondrial metabolism, redox signaling, and fusion: a mitochondria-ROS-HIF-1alpha-Kv1.5 O2-sensing pathway at the intersection of pulmonary hypertension and cancer. Am J Physiol Heart Circ Physiol 294: H570-H578, 2008.

16. Déry MA, Michaud MD and Richard DE: Hypoxia-inducible factor 1: regulation by hypoxic and non-hypoxic activators. Int J Biochem Cell Biol 37: 535-540, 2005.

17. Bonello S, Zähringer C, BelAiba RS, Djordjevic T, Hess J, Michiels C, et al: Reactive oxygen species activate the HIF-1alpha promoter via a functional NFkappaB site. Arterioscler Thromb Vasc Biol 27: 755-761, 2007.

18. McMurtry MS, Archer SL, Altieri DC, et al: Gene therapy targeting survivin selectively induces pulmonary vascular apoptosis and reverses pulmonary arterial hypertention. J Clin Invest 115: 14791491, 2005.

19. Stenmark KR, Meyrick B, Galie N, Mooi WJ and McMurtry IF: Animal models of pulmonary arterial hypertension: the hope for etiological discovery and pharmacological cure. Am J Physiol Lung Cell Mol Physiol 297: L1013-L1032, 2009.

20. Sakao S, Tatsumi K and Voelkel NF: Reversible or irreversible remodeling in pulmonary arterial hypertension. Am J Respir Cell Mol Biol 43: 629-634, 2010.

21. Okada K, Tanaka Y, Bernstein M, Zhang W, Patterson GA, and Botney MD: Pulmonary hemodynamics modify the rat pulmonary artery response to injury. A neointimal model of pulmonary hypertension. Am J Pathol 151: 1019-1025, 1997.

22. Nishimura T, Vaszar LT, Faul JL, Zhao G, Berry GJ, Shi L, et al: Simvastatin rescues rats from fatal pulmonary hypertension by inducing apoptosis of neointimal smooth muscle cells. Circulation 108: 1640-1645, 2003.

23. Faul JL, Nishimura T, Berry GJ, Benson GV, Pearl RG and Kao PN: Triptolide attenuates pulmonary arterial hypertension and neointimal formation in rats. Am J Respir Crit Care Med 162: 2252-2258, 2000.

24. Madhok BM, Yeluri S, Perry SL, Hughes TA and Jayne DG: Dichloroacetate induces apoptosis and cell-cycle arrest in colorectal cancer cells. Br J Cancer 102: 1746-1752, 2010

25. Bowers R, Cool C, Murphy RC, Tuder RM, Hopken MW, Flores SC, et al: Oxidative stress in severe pulmonary hypertension. Am J Respir Crit Care Med 169: 764-769, 2004.

26. Bonnet S, Michelakis ED, Porter CJ, Andrade-Navarro MA, Thébaud B, Haromy A, et al: An abnormal mitochondrial-hypoxia inducible factor-lalpha-Kv channel pathway disrupts oxygen sensing and triggers pulmonary arterial hypertension in fawn hooded rats: similarities to human pulmonary arterial hypertension. Circulation 113: 2630-2641, 2006.

27. Fijalkowska I, Xu W, Comhair SAA, Janocha AJ, Mavrakis LA, Krishnamachary B, et al: Hypoxia inducible-factorlalpha regulates the metabolic shift of pulmonary hypertensive endothelial cells. Am J Pathol 176: 1130-1138, 2010.

28. Dorfmüller P, Chaumais MC, Giannakouli M, Durand-Gasselin I, Raymond N, Fadel E, et al: Increased oxidative stress and severe arterial remodeling induced by permanent high-flow challenge in experimental pulmonary hypertension. Respir Res 12: 119, 2011.

29. Xu D, Guo H, Xu X, Lu Z, Fassett J, Hu X, et al: Exacerbated pulmonary arterial hypertension and right ventricular hypertrophy in animals with loss of function of extracellular superoxide dismutase. Hypertension 58: 303-309, 2011

30. Brüne B and Zhou J: Nitric oxide and superoxide: interference with hypoxic signaling. Cardiovasc Res 75: 275-282, 2007.

31. Van Rheen Z, Fattman C, Domarski S, Majka S, Klemm D, Stenmark KR, et al: Lung extracellular superoxide dismutase overexpression lessens bleomycin-induced pulmonary hypertension and vascular remodeling. Am J Respir Cell Mol Biol 44: 500-508, 2011.

32. BelAiba RS, Djordjevic T, Bonello S, Flügel D, Hess J, Kietzmann T, et al: Redox-sensitive regulation of the HIF pathway under non-hypoxic conditions in pulmonary artery smooth muscle cells. Biol Chem 385: 249-257, 2004.

33. Görlach A, Diebold I, Schini-Kerth VB, Berchner-Pfannschmidt U, Roth U, Brandes RP, et al: Thrombin activates the hypoxia-inducible factor-1 signaling pathway in vascular smooth muscle cells: Role of the p22(phox)-containing NADPH oxidase. Circ Res 89: 47-54, 2001.

34. Dahal BK, Kosanovic D, Kaulen C, Cornitescu T, Savai R, Hoffmann $\mathrm{J}$, et al: Involvement of mast cells in monocrotaline-induced pulmonary hypertension in rats. Respir Res 12: 60, 2011. 